\title{
PUF-based Mutual Authentication and Key-Exchange Protocol For Peer-to-Peer loT Applications
}

\author{
Yue Zheng, Member, IEEE, Wenye Liu, Graduate Student Member, IEEE, Chongyan Gu, Member, IEEE, \\ and Chip Hong Chang, Fellow, IEEE
}

\begin{abstract}
Peer to Peer (P2P) or direct connection loT has become increasingly popular owing to its lower latency and higher privacy compared to database-driven or server-based loT. However, wireless vulnerabilities raise severe concerns on loT device-to-device communication. This is further aggravated by the challenge to achieve lightweight direct mutual authentication and secure key exchange between loT peer nodes in P2P loT applications. Physical unclonable function (PUF) is a key enabler to lightweight, low-power and secure authentication of resource-constrained devices in loT. Nevertheless, current PUF-enabled authentication protocols, with or without the challenge-response pairs (CRPs) of each of its interlocutors stored in the verifier's side, are incompatible for P2P IoT scenarios due to the security, storage and computing power limitations of loT devices. To solve this problem, a new lightweight PUF-based mutual authentication and key-exchange protocol is proposed. It allows two resource-constrained PUF embedded endpoint devices to authenticate each other directly without the need for local storage of CRP or any private secrets, and simultaneously establish the session key for secure data exchange without resorting to public-key algorithm. The proposed protocol is evaluated using the Mao and Boyd logic as well as the automatic security analysis tool ProVerif to corroborate its mutual authenticity, secrecy, and resistance against replay and man-in-the-middle attacks. Using two Avnet Ultra96-V2 boards to emulate the two loT endpoint devices of a network, a physical prototype system is also constructed to demonstrate and validate the feasibility of the proposed secure P2P connection scheme.
\end{abstract}

Index Terms-Peer-to-peer Internet of Things, loT Security, Physical Unclonable Functions, Peer-entity Authentication Protocol, Key Exchange Protocol, Man-in-the-Middle Attacks

\section{INTRODUCTION}

$\mathrm{T}$ HE Internet of Things (IoT) regime can be roughly classified into two categories, i.e., database-driven IoT and peer-to-peer (P2P) IoT [1]. For database-driven IoT, the connections between two IoT endpoint devices are established indirectly through a server, which is a common approach for IoT connection. Data collected from the IoT devices will be transmitted to and stored in the server's database and the retrieval of data can be achieved by sending a request to the server. By contrast, P2P IoT enables direct connection between two IoT endpoints, which provides lower-latency performance and higher-privacy level compared to server-centric database-driven IoT. As data collectors, IoT endpoints have access to high-value assets and confidential information. Since they are easily accessible and resource constrained, they are also alluring targets of attack, especially when the intelligent autonomous endpoint devices are allowed to interact or exchange sensitive data directly with each other endpoints in a smart environment. Authentication and secure key exchange between two end-

- Y. Zheng, W. Liu and C.H. Chang are with the School of Electrical and Electronic Engineering, Nanyang Technological University, Singapore. E-mail:yue.zheng@ntu.edu.sg,wliu015@e.ntu.edu.sg, echchang@ntu.edu.sg

- C. Gu is with the Centre for Secure Information Technologies (CSIT), Institute of Electronics, Communications and Information Technology (ECIT), Queens University Belfast (QUB), U.K., BT3 9DT.

E-mail: C.Gu@qub.ac.uk

Manuscript received Mar. 4, 2021. points are the crucial first line of defense to prevent fake endpoints from exfiltrating data and holding information hostage. Unfortunately, legacy security solutions, despite sufficiently mature, do not transfer convincingly to the P2P IoT scenarios due to the heterogeneity, and resource and memory constraints of IoT devices.

To this end, Physical Unclonable Function (PUF) stands out as a promising embodiment to facilitate secure authentication and key exchange of IoT devices [2], [3], [4], [5], [6]. A PUF can be viewed as a physical circuit realization of a random oracle by harnessing the minute variance in modern semiconductor manufacturing processes [7]. The lynchpin of PUF is the irreversible random mapping of a digital input (known as a challenge) to a digital output (known as a response). The challenge-response mapping is very similar to a cryptographic hash function except that the hardness to invert the function is originated from the physical disorder instead of the computational complexity theory. The chip-tochip variances of a manufacturing process can be harvested by the PUF circuit. With enough basic PUF cells, many unique challenge-response pairs (CRPs) of arbitrary length can be generated from each chip for a huge number of manufactured chips. Although the PUF circuit itself is easy to make and the responses can be readily measured, it is practically infeasible to physically clone a PUF instance to reproduce the same CRPs. More importantly, no secret key needs to be stored locally on a device as the PUF can generate the device-specific secret (response) only upon request 
(by applying a challenge). Once the required number of CRPs have been successfully measured and enrolled into a secure server database, the external measurement interface of the PUF responses can be permanently disabled. Since the entire set of CRPs is intricately embodied in the nanostructure of the PUF, any active manipulation of the PUF circuit internals will cause dysfunction of the challengeresponse mapping mechanism and destroy the secret. This tamper-evident property of PUF and its ability to securely identify a device by interrogation without the need for a permanent secret residence in anti-temper memory largely reduce the risks of a number of powerful hardware attack vectors such as reverse engineering, probing and fault injection attacks on seizable devices [8], [9], [10], [11].

Using PUF for device authentication requires the verifier to keep a copy of its prover's CRPs. This is a common practice in PUF-based authentication protocol in databasedriven IoT as the server has the computing power and memory resources to securely store the database of the CRPs of all its interlocutors. However, this requirement becomes impractical when mutual authentication has to be performed directly between two end-devices for secure communication in P2P IoT applications. IoT end-devices are usually small, low cost and resource constrained, which render classical recipes [2], [5], [6] of PUF-based mutual authentication between device and server inapplicable, especially when one end-device needs to communicate with several other end-devices. Besides, storing the prover's CRPs in the verifier device allows the latter to impersonate the prover, which rules out the applicability of traditional PUF-based authentication scheme in the P2P IoT scenarios.

This paper presents a lightweight device-to-device PUFbased authentication protocol between IoT edge devices for P2P IoT connection without requiring the devices to store each other's CRPs. The concept was firstly proposed without implementation in our preliminary work [12]. That work still requires the server's participation during the authentication phase and is vulnerable to the two-time pad attack. The shortcomings and overly simplistic security analysis in [12] are addressed in this paper. Our major contributions are:

1 The proposed mutual authentication scheme enables secure direct communication between any two IoT endpoints in a network. This enables the IoT data to be shared directly between a pair of endpoints. The server only helps to create the connection in the enrolment and update phase, and is then completely released from the authentication and communication phase of the system.

2 The proposed PUF-based scheme requires no keys or CRPs to be stored in the IoT endpoints. No extra intermediate "verifier" node is required, which is unlike the scheme in [13]. This makes it possible for a resource-constrained PUF-embedded device to directly authenticate any other PUF-embedded devices in the same local area network.

3 The proposed scheme also achieves a secure key exchange as a byproduct of the protocol. The session keys for exchanging messages by symmetric-key cryptography between two devices are directly derived from their PUF responses. The shared keys are automatically established upon successful authentication, which of- floads the complexity of managing and storing the keys for IoT devices.

4 The security of the protocol is formally verified using the Mao and Boyd Logic [14] and the professional security verification tool ProVerif [15]. The proposed scheme is proved to possess robust reachability and secrecy, mutual authenticity, and high resilience against multiple attacks such as replay and man-in-the-middle attacks.

5 The proposed scheme has also been evaluated physically by a prototype system using Avnet Ultra96-V2 boards to emulate the IoT endpoints equipped with the required hardware primitive. Hardware overhead, computational complexity and other system performance are analyzed and compared with related existing works.

The rest of the paper is organized as follows. Sec. 2 introduces the related works and preliminaries; Sec. 3 presented the proposed protocol design, which includes an enrolment and update phase, and a mutual authentication and key exchange phase. The security of the protocol is analyzed in Sec. 4 while the implementation and comparison are presented in Sec. 5. Finally, Sec. 6 concludes the paper.

\section{Related Works and Preliminaries}

In this section, two pitfalls in using traditional authentication protocols for securing P2P IoT applications are discussed. In the same light, the limitations of existing PUFbased device authentication schemes in IoT setting are also identified. In addition, inference rules of Mao and Boyd Logic that can be used for the formal security analysis of P2P IoT device authentication protocol are introduced.

\subsection{P2P loT}

Two necessary steps and the potential issues of adopting typical authentication protocols for securing P2P IoT connection are identified as follows. Firstly, the authenticity of the two IoT endpoints has to be assured before a secure direct connection between them can be established. A common approach is to involve a trusted server to authenticate the two IoT devices individually. Once both devices are authenticated, the communication is then handed over to the two devices without involving the server any more. This approach requires two mutual authentications to be performed independently between the server and the devices. The extra authentication through a proxy server incurs transmission bandwidth overhead and authentication delay owing to the need to protect any sensitive secret that cannot be sent in plaintext. Besides, the establishment and relay of shared secret through the server for each authentication request also increase the vulnerability of man-in-the-middle (MITM) and chosen-plaintext or chosen-ciphertext attacks. Secondly, end-to-end encryption is required to ensure secure data transmission between the two interlocutors. Either a symmetric key or a public key cryptographic (PKC) algorithm can be utilized for data encryption. Both cases require a secret key to be safely stored in both endpoints. If the secret keys are stored in the non-volatile memory (NVM) of the inexpensive endpoint devices with limited room for 
sophisticated key protection, they can be relatively easily extracted by powerful commercial data extraction tools like Graykey [16] of Grayshift and UFED [17] of Cellebrite.

The abovementioned issues of P2P IoT can be avoided if the two IoT endpoints can directly authenticate each other without the need to store the secret key persistently and locally on the endpoint device to achieve end-to-end encryption.

\subsection{Existing PUF-based Authentication Schemes}

PUF as a "device fingerprint" has been widely used for individual device authentication [2], [5], [6]. A typical PUFbased authentication process is shown in Fig. 1. An enrolment phase is required to be carried out securely in a controlled environment once before the PUF can be deployed in the field for device authentication. During the enrolment phase, an adequate number of CRPs of the PUF has to be collected and stored in the verifier's database. In the authentication phase, the verifier randomly selects a CRP from its database and then transmits the challenge to the prover. The prover obtains a response by applying this challenge to its PUF and sends the response back to the verifier. By comparing the received PUF response with the enrolled one, the verifier can authenticate the prover's device.

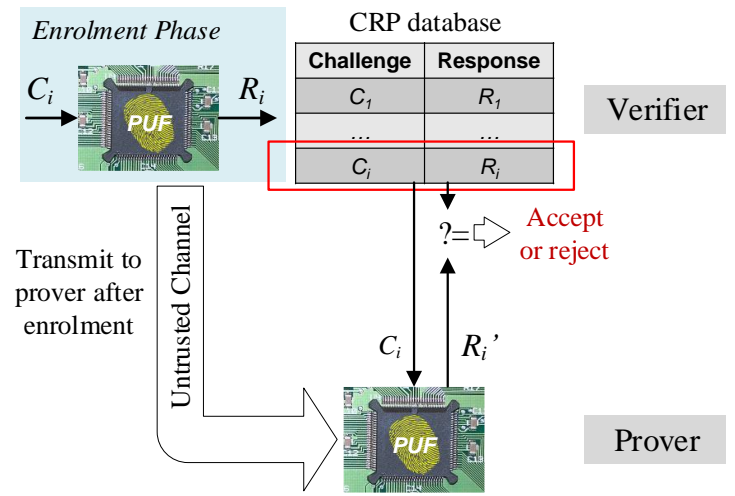

Fig. 1. A typical PUF-based device authentication scheme. Here the PUF is a strong PUF with exponential number of CRPs. Each CRP is used only once for secure authentication.

As shown from Fig. 1, a typical PUF-based authentication scheme requires the verifier to safely store a CRP database of the prover's PUF. This is easier to achieve if the verifier is a powerful server. If direct authentication of P2P IoT devices is to be achieved without a proxy server as discussed in Sec. 2.1, this scheme is infeasible as the verifier, an IoT endpoint, has limited resources to ensure the secure storage of the CRPs. As a result, PUF-based authentication schemes without the CRP storage in both endpoint devices of the verifier and the prover are desperately needed in such P2P IoT applications.

Several relevant solutions have been proposed in the literature [13], [18], [19], [20]. They are briefly reviewed as follows.

The protocol of [13] combines the PUF with a certificateless identity based encryption. It requires a verifier node to act as the proxy to authenticate the two IoT endpoints. It also requires the presence of a Security Association
Provider (SAP) during the authentication phase to manage the authentication helper data. Besides, the protocol requires the verifier to store a secret hash key in an NVM. This requirement has completely defeated the fundamental tenet of using PUF to avoid keeping the secret in local memory.

On the other hand, the Babel-chain PUF-based mutual authentication protocol (BC-PHEMAP) [18] applies the PUF response iteratively to the PUF to create PUF chains for authentication. Since multiple PUF operations (8 in the text example) are required and each PUF operation has to be $100 \%$ reliable in order to preserve the enrolled chain result, this scheme is not practical due to the inevitable unreliability of PUF responses in the field. What is more, the BC-PHEMAP scheme reveals raw PUF responses during the protocol executions. The scheme requires strong PUF to ensure the chain or its subset is never reused to pair different devices but this also provides an opportunity for the adversary to collect a large number of used raw responses for modelling attack.

The scheme in [19] also requires a trusted third party server during the mutual authentication of two IoT endpoints. The server is responsible for providing the authentication message and generating the session keys. The protocol trades message exchange times (delays) for zerostorage in the device side and reduced storage requirement in the server side. Two rounds of mutual authentication between the server and the device and one round of mutual authentication between devices are required for a complete protocol run. On a par with [18], it does not take into account the practical PUF reliability issues.

Similar to [13], the work [20] relies on the combination of PUF with a PKC on elliptic curve to achieve mutual authentication between IoT nodes without the need for a local CRP storage. Each IoT node will obtain its public and private key pair after an enrolment phase with the server in a secure environment. The public keys are stored in the device's memory while the private keys are recovered with the help of the PUF during the authentication phase. 14 rounds of elliptic curve scalar multiplications are required to complete the protocol runs, which increases the computational cost of the protocol significantly.

In short, existing PUF-based authentication schemes, either with or without requiring a CRP storage in the verifier, cannot be used to instantiate a low-cost, key-less, directly authenticated and directly connected P2P IoT network.

\subsection{The Mao and Boyd Logic}

The Mao and Boyd (MB) logic [14] is a formal logical approach towards the security analysis of peer-entity authentication protocols. It adopts the basic notational framework of the Burrows, Abadi and Needham (BAN) logic [21], but has overcome its limitations, such as providing only partial correctness proof and lacking complete formality. This section provides a brief introduction of the MB Logic.

Table 1 lists some basic logical formulas. In this table, $P$ with or without any subscript represents a principal, $M$ with or without any subscript represents a message and $F$ with or without any subscript represents a formula. $K$ represents a secret key, which is also a type of message.

The first step to analyze a protocol using the MB logic is to perform a protocol message idealization. Message 
TABLE 1

Logical Formulas in the MB logic and Their Explanations

\begin{tabular}{|c|c|}
\hline Formulas & Explanations \\
\hline$M_{1} \Re M_{2}, M_{1} \mid M_{2}$ & $\begin{array}{c}\text { Combined message; } \Re \text { combines different types of } \\
\text { messages while } \mid \text { combines the same type of } \\
\text { messages }\end{array}$ \\
\hline$F_{1} \wedge F_{2}$ & Boolean conjunction of sub-formulas $F_{1}$ and $F_{2}$ \\
\hline$P \mid \equiv F$ & $P$ believes the correctness of $F$ \\
\hline$P \stackrel{K}{\sim} M$ & $P$ send out $K$-encrypted $M$ \\
\hline$P \stackrel{K}{\triangleleft} M$ & $P$ sees $M$ by using the decipherment key $K$ \\
\hline$P_{1} \stackrel{K}{\longleftrightarrow} P_{2}$ & $K$ is a good shared key between $P_{1}$ and $P_{2}$ \\
\hline $\sup (P)$ & $\begin{array}{c}P \text { is a super-principal who has to be trusted } \\
\text { unconditionally. IoT devices can be a } \\
\text { super-principal for its PUF CRPs and the nonces it } \\
\text { generated. }\end{array}$ \\
\hline$\#(M)$ & $M$ is fresh \\
\hline$S^{c} \triangleleft \| M$ & $\begin{array}{l}\text { Those principals that in the complement set of the } \\
\text { set } S \text { cannot see } M\end{array}$ \\
\hline
\end{tabular}

idealization interprets the implicit protocol messages and transcribes them into explicit specifications. The following formalized terms are defined and used in the idealization rules:

1) Atomic message: a message without any ",", “|", “ $R^{\prime \prime}$, "(", ")", " $\{$ ", “ $\}$ " appears in its construction.

2) Challenge: an atomic message sent or received in different protocol lines by the same principal (i.e., the originator, which can be a person, a server, or a device). A timestamp is not considered as a challenge.

3) Replied challenge: a challenge sent to its originator.

4) Response: an atomic message that appears in a protocol line with a replied challenge and itself sent together by the sender of that line. A timestamp is not considered as a response.

5) Nonsense: an atomic message if it is not a challenge, a response or a timestamp.

With the definitions of the above formalized terms, the rules for idealization can be described as follows:

1) Delete any nonsense.

2) Treat an atomic message as response if it appears in a line not only as a challenge but also as a response.

3) Use the operator "|" to combine multiple factors in a line that belongs to the same type, e.g., "challenge | challenge", "response 1 response".

4) Use the operator " $\Re$ " to combine multiple factors in a line that belong to different types, e.g., "response $\Re$ replied challenge", "message $\Re$ timestamp".

Next, a set of inference rules or the logics of belief are defined for reasoning about the beliefs of principals and logical formulas. For simplicity, only the inference rules that are related to and used in this work are introduced.

1) The authentication rule:

if $P$ and $Q$ share a good key $K$, and $P$ can see $M$ using the key $K$, then $P$ can believe $Q$ has sent out the $K$ - encrypted $M$.

$$
\frac{P \mid \equiv P \stackrel{K}{\longleftrightarrow} Q \wedge P \stackrel{K}{\triangleleft} M}{P|\equiv Q| \sim M}
$$

2) The confidentiality rule:

if $P$ and $Q$ share a good key $K$, and $P$ believes that only the principles in the set $S$ is able to see $M$, and $P$ has sent out the $K$-encrypted $M$, then $P$ can believe that $M$ is available to only the set $S$ and $Q$ except itself.

$$
\frac{P|\equiv P \stackrel{K}{\longleftrightarrow} Q \bigwedge P| \equiv S^{c} \triangleleft \| M \bigwedge P \mid \sim M}{\stackrel{K}{\longrightarrow}}
$$

3) The nonce-verification rule:

If $P$ believes that $M$ is fresh and $P$ believes that $Q$ has sent out the $K$-encrypted $M$, then $P$ can believe that $Q$ believes $K$ is a good shared key between $P$ and $Q$.

$$
\frac{P|\equiv \#(M) \wedge P| \equiv Q \mid \sim M}{\stackrel{K}{\sim} M}
$$

4) The super-principal rule:

If $P$ believes that $Q$ is a super principal, then $P$ believes what $Q$ believes.

$$
\frac{P|\equiv Q| \equiv X \bigwedge P \mid \equiv \sup (Q)}{P \mid \equiv X}
$$

5) The fresh rule:

$P$ can believe that $N$ is fresh if he receives $N$ with a fresh $M$ together in a message.

$$
\frac{P \mid \equiv \#(M) \wedge P \triangleleft N \Re M}{P \mid \equiv \#(N)}
$$

6) The good-key rule:

If $P$ believes that $K$ is fresh and available only to $P$ and $Q$, then $P$ can believe that $K$ is a good shared key between $P$ and $Q$.

$$
\frac{P\left|\equiv\{P, Q\}^{c} \triangleleft \| K \bigwedge P\right| \equiv \#(K)}{P \mid \equiv P \stackrel{K}{\longleftrightarrow} Q}
$$

7) The derived rule:

By combining the following belief axiom

$$
\frac{P|\equiv X \bigwedge P| \equiv Y}{P \mid \equiv(X \bigwedge Y)}
$$

with the confidentiality rule (2), a derived rule can be obtained as follows:

$$
\frac{P|\equiv Q| \equiv Q \stackrel{K}{\longleftrightarrow} P \wedge P|\equiv Q| \equiv S^{c} \triangleleft \| M \wedge P|\equiv Q| \stackrel{K}{\sim} M}{P|\equiv Q| \equiv(S \cup\{P\})^{c} \triangleleft \| M}
$$

Finally, the pre-conditions (initial beliefs) of a security protocol can be obtained by reversely applying the inference rules to the expected protocol achievements. If the preconditions obtained are reasonable or is satisfiable by a protocol run, then the protocol is construed to be provably secure based on the logical theory of belief (doxastic logic). 


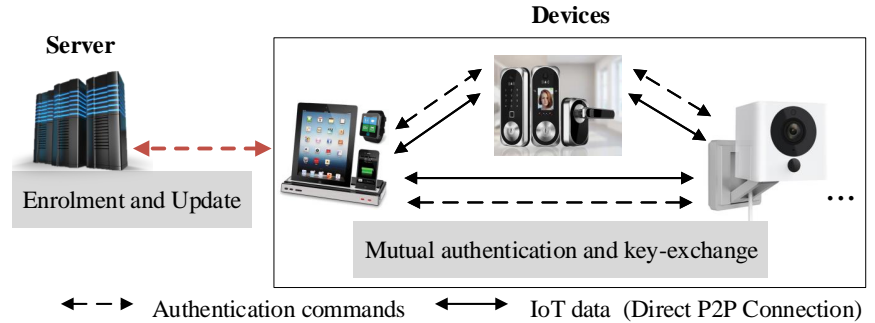

Fig. 2. System model. The server is only present in the enrolment and update phase to create/update the authentication mask and helper data. Once this is completed, the loT endpoints can freely and directly authenticate and exchange data among themselves.

\section{Proposed PUF-based Scheme}

\subsection{Models and Assumptions}

Fig. 2 shows the system model. The objective of the system is to establish direct mutual authentication and direct communications between IoT peer nodes by utilizing the on-the-fly key generation property of PUF while avoiding the shortfalls of existing PUF-based authentication schemes. The end-devices are assumed to be embedded with a PUF, a lightweight hash function (for example, sponge-like construction) [22] and a lightweight block cipher optimized for performance in hardware implementation like SIMON [23]. We assume that the CRPs of the devices have already been securely collected in the design house and securely transmitted to a legal local server. The end-devices have limited storage capacity and are incapable of protecting the secrets stored in their NVM. In contrast, the server can afford expensive anti-tamper storage to secure any secret and is powerful enough to perform complex computations.

The threat model assumes the presence of an attacker who can connect to the network and is able to eavesdrop the communication channel during the authentication process. It is also assumed that the attacker can initiate an unbound number of authentication requests. The attacker cannot break into the server's database but can have access to an IoT end-device to retrieve any stored secrets in the device. The objective of the attacker is to impersonate any end-devices and steal the secret session key without being detected.

The proposed protocol consists of two phases, i.e., an enrolment and update phase, and a mutual authentication and key-exchange phase. As shown in Fig. 2, the IoT enddevices only have to communicate with the server in the enrolment and update phase. After which, the IoT peer nodes can connect and authenticate each other directly. The notations used in our proposed protocol are described in Table 2.

\subsection{Enrolment and Update Phase}

The enrolment phase is firstly conducted in the design house, where the CRPs of each PUF-embedded chip are collected after fabrication. After which, the direct CRP access path are permanently removed. Once the customer identity is authenticated, the CRPs will be transferred to the customer's local server for the intended applications. Note that the CRP collection is assumed to be conducted
TABLE 2

Notations used by the proposed protocol

\begin{tabular}{c|c}
\hline Notation & Description \\
\hline$P U F(C)$ & Apply the challenge $C$ to the PUF \\
\hline$H(X)$ & Hash of $X$ \\
\hline$\oplus$ & Exclusive OR \\
\hline$\|$ & Concatenation \\
\hline$E N C(X, k)$ & Encrypt $X$ using the key $k$ \\
\hline$D E C(X, k)$ & Decrypt $X$ using the key $K$ \\
\hline$\phi$ & $\begin{array}{c}\text { Arror correction code (ECC) generation } \\
\text { phase. It generates the helper data } h \text { for the } \\
\text { recovery of } X\end{array}$ \\
\hline ECC_gen $(X)$ & $\begin{array}{c}\text { ECC reproduction phase. It corrects the } \\
\text { noisy } X \text { with the helper data } h\end{array}$ \\
\hline$E C C \_r e p(X, h)$ &
\end{tabular}

in a secure environment and the transfer of CRPs is also securely completed with the help of various supply chain authentication protocols.

The main idea is to utilize a one-time pad (OTP) to generate a key mask for a dedicated device pair. The mask can be publicly stored in the device side without compromising the security. Its purpose is to help with the retrieval of the secrets during the authentication process. Specifically, as shown in Fig. 3, for each intended pair of edge devices in an IoT network, $A$ and $B$ for example, a challenge $C_{A, B}$ is generated. The corresponding responses, $R_{A}$ and $R_{B}$, can be retrieved from the server's CRP database for $A$ and $B$ accordingly. Helper data $h_{A}$ and $h_{B}$ are generated by the ECC [24] for the recovery of $R_{A}$ and $R_{B}$, respectively, from the noisy responses reproduced by the PUF in the field. The server calculates a pair of random intermediary secrets, $P_{1}$ and $P_{2}$, from the response $R$ of each device. A lightweight cryptographic hash function $H$ is used to boost the entropy of PUF responses and prevent the direct use of raw responses. Then, a OTP mask $\phi$ is generated by the XOR operation. $\phi$ needs not be kept private and it can be stored in the device memory. Due to the OTP, the knowledge of $\phi$ does not help to reveal $P_{1}$ and $P_{2}$. Hence, both $P_{1}$ and $P_{2}$ can serve as the keys to decrypt a pair of secrets designated for a device with the help of its PUF. Specifically, for device $A, P_{1 A}$ is first calculated by hashing its reproduced PUF response $R_{A} . P_{2 A}$ is then obtained by hashing $P_{1 A} . P_{1 B}$ and $P_{2 B}$ are similarly obtained from hashing $R_{B}$ of device $B$ consecutively. These private data are concealed in $\phi_{1}$ and $\phi_{2}$ by the OTP construction, $P_{1 A} \oplus P_{1 B}$ and $P_{2 A} \oplus P_{2 B}$, respectively.

Note that a OTP can achieve perfect secrecy, if the length of the random key is at least as long as the plaintext. Besides, the random key must be kept completely secret and never be reused in whole or in part. Most of the requirements can be easily achieved in our proposed scheme as the random keys (or plaintexts) defined by the PUF response can be generated by the device when needed. However, the one-time use policy of OTP requires that "a device should 
never be paired with multiple other edge devices for the same challenge". Assume that there are $N$ devices in the network and each device can pair with any other device to form a direct communication pair. To avoid the same challenge from being used in multiple pairs involving the same device, the challenges are allocated at the server side by Algorithm 1 , where $I_{i, j}$ denotes the index of the challenge $C_{i, j}$ used for the paired devices, $i$ and $j$. After running Algorithm 1 for a specific application, the server is able to randomly generate $M$ number of challenges and allocates them to each device pair according to the challenge index allocation list $C L$.

A device should only accept the authentication mask from an authorized server that possesses the correct PUF CRPs of the device. To ensure this, the server packs the mask $\phi_{2}$ with the key $P_{1 A}$, a timestamp $T$ and the device $B^{\prime}$ s identity $I D_{B}$ together. The message package is encrypted with the key $P_{1 A}$. The encrypted message is concatenated with the challenge $C_{A, B}$ and the helper data $h_{A}$ to form a message $M_{A}$ for sending to the device $A$. Upon receiving $M_{A}$, the device $A$ applies $C_{A, B}$ to generate the key $\tilde{P}_{1 A}$. If the key is correct, the message can be successfully decrypted. If the retrieved timestamp is fresh and the decrypted $P_{1 A}$ matches $\tilde{P}_{1 A}$, the device $A$ can believe the enrolment or updated message $\left(I_{B}, C_{A, B}, h_{A}, \phi_{2}\right)$ is indeed from a trusted local server. The timestamp $T$ in $M_{A}$ and $M_{B}$ ensures the freshness of the message and prevents the replay of a compromised $M_{A}$ or $M_{B}$. Each edge device can store $N-1$ masks if it would like to establish a secure authenticated connection with all other devices in the network. The same process can be performed whenever the mask data in the device's side need to be updated. Due to the allocation of unique challenges to device pairs, although the authorized device can retrieve the hashed PUF response to the allocated challenge for any of its interlocutor, it still cannot impersonate as its interlocutor to communicate with other devices.

\subsection{Mutual Authentication and Key-Exchange Phase}

If an IoT end-device $A$ wants to communicate with another end-device $B$, it will send a request message containing the current timestamp $T_{1}$ and its identity $I D_{A}$ to the device $B$, as shown in Fig. 4. $B$ checks the identity of $A$ in its database. If it exists, it fetches the enrolled challenge $C_{A, B}$, helper data $h_{B}$ and mask $\phi_{1}$ corresponding to $A$ from its memory. $B$ applies the challenge $C_{A, B}$ to its embedded PUF to generate a reliable PUF response with the help data $h_{B}$. Using the mask $\phi_{1}, B$ is able to recover the hashed PUF response $\tilde{P}_{1 A}$ of $A$ to the challenge $C_{A, B}$ by XORing $\phi_{1}$ with $P_{1 B}$. The latter is the hash of $R_{B}$. B then generates a nonce $n_{A}$ that satisfies $n_{A} \neq \tilde{P}_{1 A}$. A plaintext $m_{A}$ is generated for $A$ by XORing $n_{A}$ with $P_{1 B}$. Using the recovered $\tilde{P}_{1 A}$ as the encryption key, a puzzle $Q_{A}$ is created by the device $B$ for the device $A$ by encrypting the concatenated message of $m_{A}$ and the received $T_{1}$. To decrypt the received $Q_{A}, A$ calculates $P_{1 A}$ directly from its clean PUF response $R_{A}$ reproduced with the help of $h_{A}$. If $\tilde{T}_{1}$ obtained from the decrypted $Q_{A}$ is equal to $T_{1}, A$ will hash the recovered plaintext $\tilde{m}_{A}$ to $\tilde{V}_{A}$ and send it to $B$ as a proof of successfully solving the puzzle $Q_{A}$. Meantime, $B$ also calculates $V_{A}$ from the hashed $m_{A}$ and compares it with the received $\tilde{V}_{A}$. If they match, $B$ validates

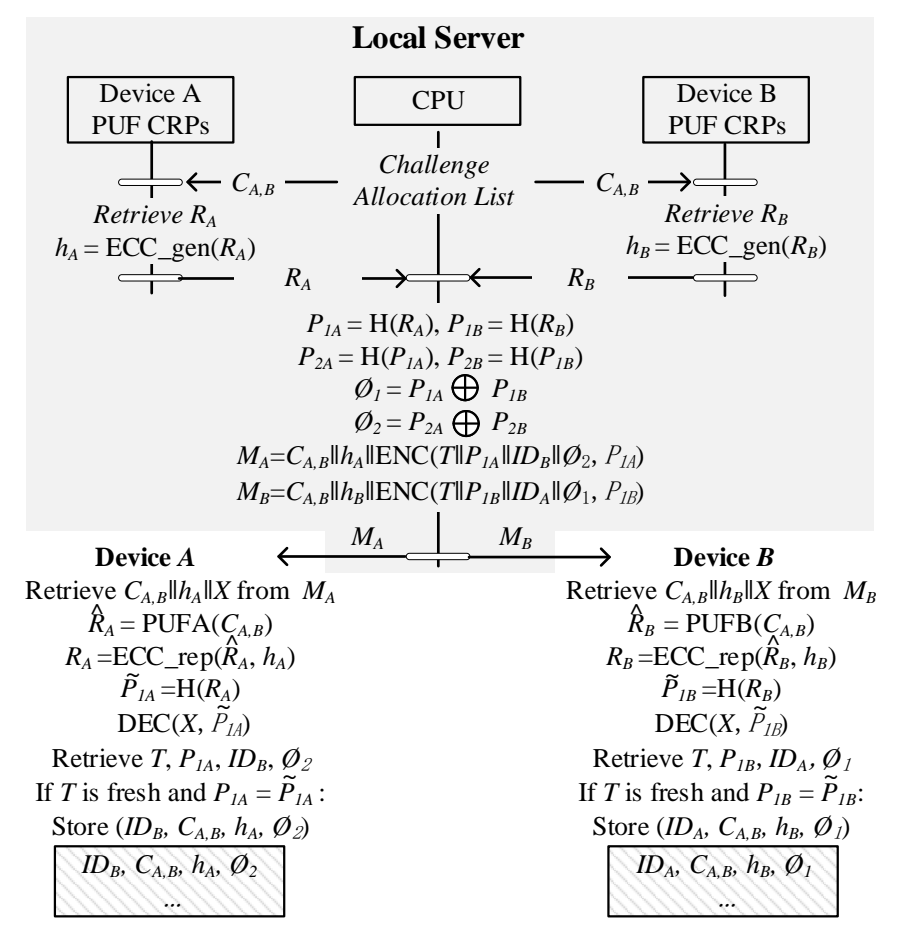

Fig. 3. Enrolment and update phase. The device only accepts the authentication mask data from the server. Here, the hat on a response denotes the response is noisy while the tilde on a variable is used to emphasize that the private variable is recovered at the device side instead of being received by a transmission. Similarly for Fig. 4 .

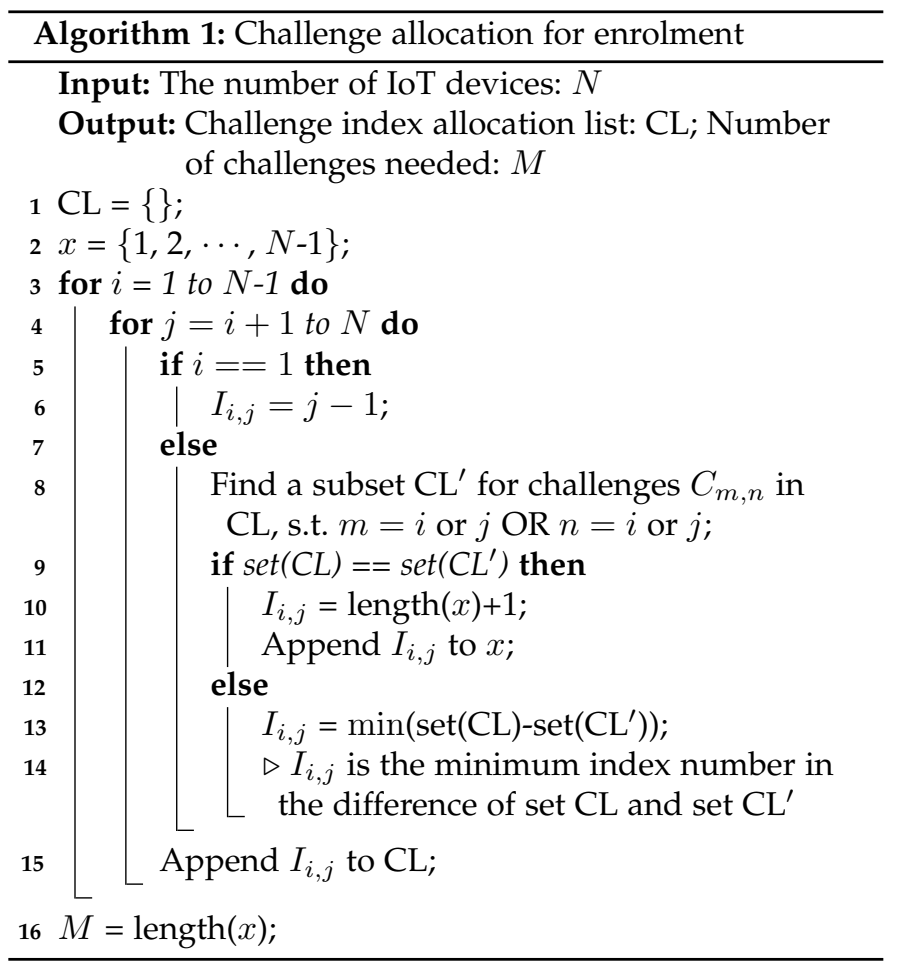

that the protocol initiator is indeed the authentic device $A$ as it is claims.

By reversing the roles of the prover and the verifier, similar flow can be reciprocated by the initiator to validate if the interlocutor is indeed the one it wants to communicate with. The device $A$ retrieves $\tilde{P}_{2 B}$ with the help of $\phi_{2}$. Using 


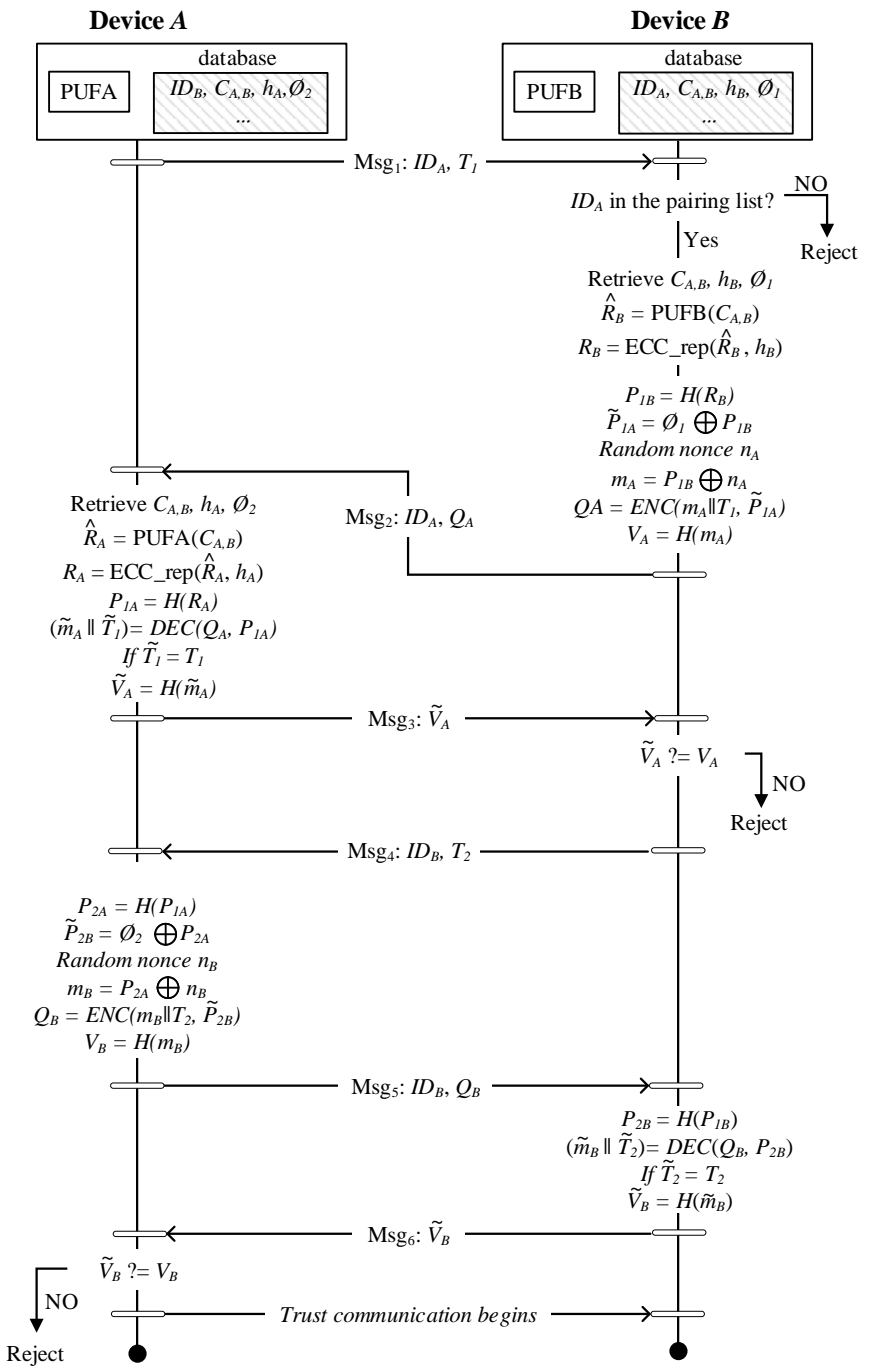

Fig. 4. Mutual authentication and key-exchange process. After the successful mutual authentication, $P_{2 A}$ and $P_{2 B}$ can be used as the session keys for message encryption by the devices, $A$ and $B$, respectively $\left(n_{A} \neq \tilde{P}_{1 A}\right.$ and $\left.n_{B} \neq \tilde{P}_{2 B}\right)$.

$\tilde{P}_{2 B}$ as a key, a message $m_{B}$ and $T_{2}$ is encrypted into a puzzle $Q_{B}$ for the device $B$ to solve. Following the same procedure, $B$ can decrypt the received ciphertext $Q_{B}$ using $P_{2 B}$ obtained by hashing its PUF response $R_{B}$ twice. The recovered plaintext $\tilde{m}_{B}$ is hashed to generate a proof $\tilde{V}_{B}$. The proof is sent to $A$ for verification. $A$ compares its received $\tilde{V}_{B}$ with its generated $V_{B}$ to complete the mutual authentication between them. Upon successful mutual authentication, $A$ can use $P_{2 A}$ as the session key to send encrypted message to $B$ and $B$ can use $P_{2 B}$ as the session key to send encrypted message to $A$. This is because the device $B$ can calculate $P_{2 A}$ by hashing its recovered $\tilde{P}_{1 A}$ and uses it to decrypt the message sent by the device $A$. Vice versa, $A$ can also calculate $P_{2 B}$ to decrypt the message sent by $B$.

\section{Security Analysis}

In this section, the MB logic introduced in Sec. 2.3 and an automatic security verification tool ProVerif [15] are used to formally analyze the security of the proposed protocol. The formal security analysis is based on some assumptions about the properties of the security primitives discussed below.

Firstly, the PUF is assumed to be secure against microprobing and physical attacks. This is because the key is not permanently present in digital form like the NVM key. It is derived by the PUF within the hardware only whenever it is required. The CRP generation mechanism cannot be derived by invasive attack or probing into the internal cell structure of the PUF. Doing so will leave a permanent tamper evidence and destroy the PUF functionality, which can be detected easily. As a natural byproduct of the semiconductor manufacturing process variations, the CRPs of the PUF are assumed to have a very high entropy. Although its generation mechanism is inexpensive, such physical disorder is the very hard to be fully characterized. In other words, it is practically infeasible for the adversary to physically clone a PUF, nor guess an unseen response of a PUF by any brute-force approach if the bitlength of the PUF response is sufficiently long. Besides, we assume that the PUF can also be made resilient against side channel attacks using several countermeasures proposed in the literature [25], [26].

Secondly, the hash function used is assumed to be a one-way hash function that possesses perfect pre-image and collision resistances. Pre-image resistance refers to the difficulty of finding any correct input given the output of the hash function, whereas collision resistance refers to the computational complexity of finding two different inputs that hash to the same output value. It should be noted that collision resistance also implies second pre-image resistance. The latter is a property of a hash function to ensure that given an input, it is difficult to find another input that has the same hash output.

\subsection{The MB logic Analysis}

Upon device deployment, the objective of the enrolment or update phase is to generate authentication mask data $\phi$ for the device pairs. The MB logic can be used to prove the authenticity and freshness of $\phi$. Taking the enrolment/update of the device $A$ for example, the idealized protocol statement is formally described as follows:

$$
S \rightarrow A: C_{A, B} \Re E N C\left(T \Re P_{1 A} \Re I D_{B} \Re \phi_{2}, P_{1 A}\right)
$$

$\phi_{2}$ can be proved to be a good shared key between the server $S$ and $A$ after the enrolment and update session: $S\left|\equiv A \stackrel{\phi_{2}}{\longleftrightarrow} S, A\right| \equiv A \stackrel{\phi_{2}}{\longleftrightarrow} S$. As shown in the tableaux of Figs. 5 (a) and (b), to prove the correctness of $S \mid \equiv A \stackrel{\phi_{2}}{\longleftrightarrow} S$, the good key rule (6) can be applied first, which leads to two pre-conditions, $S \mid \equiv\{A, S\}^{c} \triangleleft \| \phi_{2}$ and $S \mid \equiv \#\left(\phi_{2}\right)$. Since the protocol is meant for the server to enrol or update the device with a new $\phi_{2}, S \mid \equiv \#\left(\phi_{2}\right)$ is reasonable. As for the other pre-condition $S \mid \equiv\{A, S\}^{c} \triangleleft \| \phi_{2}$, the confidentiality rule (2) can be used and the three resulting assumptions are: 1) $S \mid \equiv A \stackrel{P_{1 A}}{\longleftrightarrow} S$; 2) $S \mid \equiv\{S\}^{C} \triangleleft \| \phi_{2}$; and 3) $S \mid \sim P_{1 A}$. All the three assumptions are reasonable as the server stores the correct CRP of the device and it indeed transmits its generated $\phi_{2}$ in an encrypted form to $A$ as indicated in the idealized message. Therefore, the correctness of $S \mid \equiv A \stackrel{\phi_{2}}{\longleftrightarrow} S$ is proved. Similar analysis can be conducted to show the correctness of $A \mid \equiv A \stackrel{\phi_{2}}{\longleftrightarrow} S$. Note 


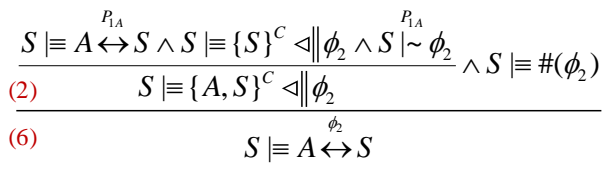

(a)

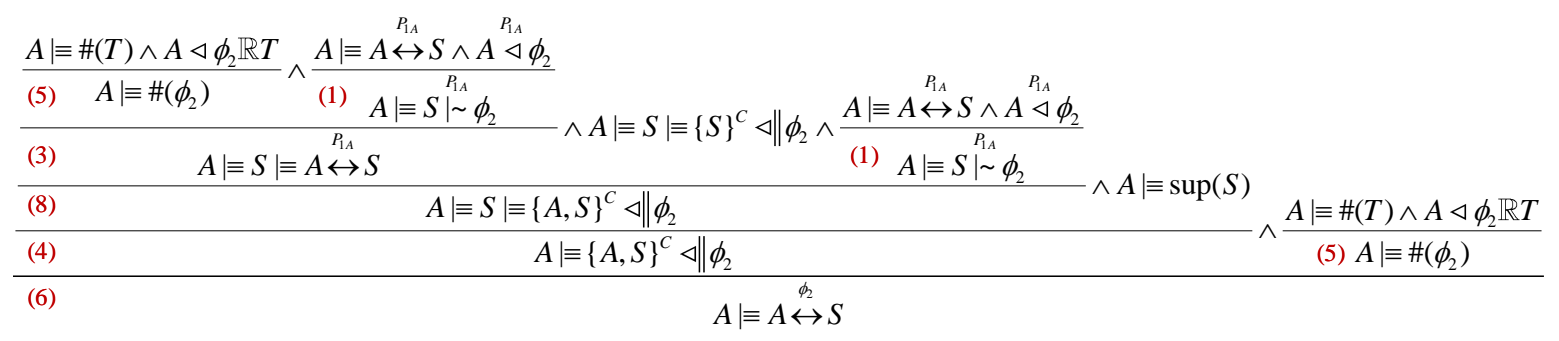

(b)

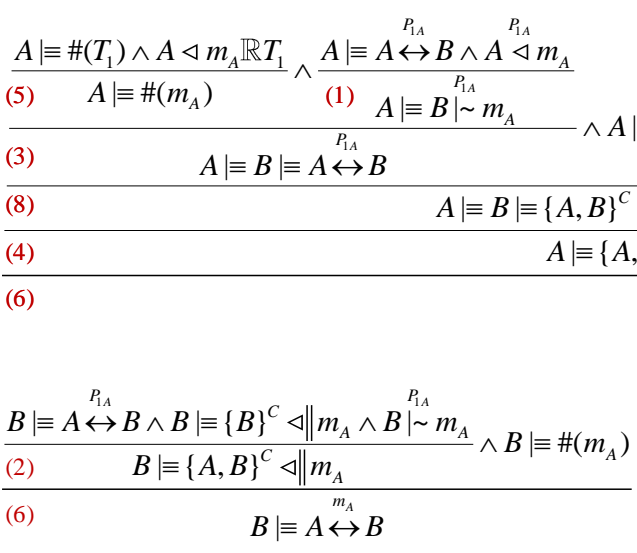

(d)

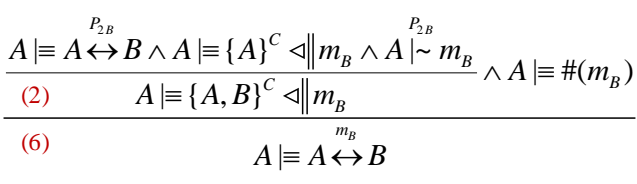

(e)

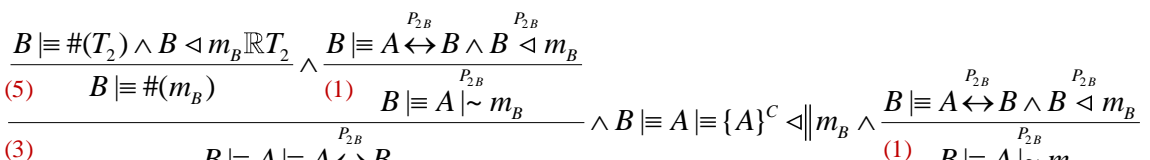

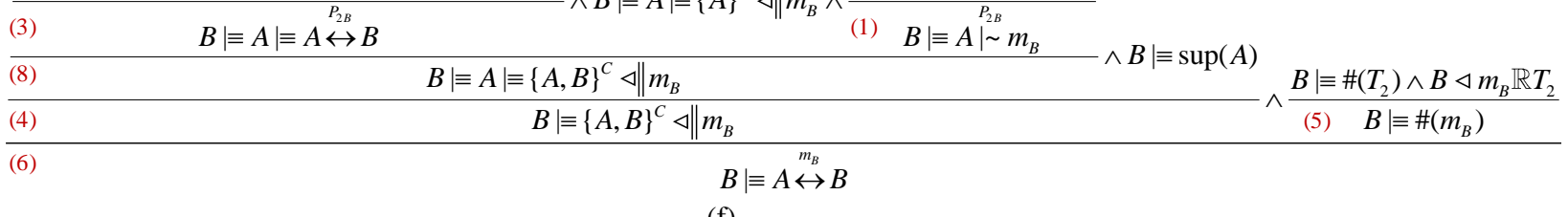

(f)

(1): The authentication rule; (2): The confidentiality rule; (3): The nonce-verification rule; (4): The super-principal rule;

(5): The fresh rule; (6): The good-key rule; (8): The derived rule.

Fig. 5. The Mao and Boyd logic based security proof of (a) $S$ believes $\phi_{2 B}$ is only available to $A$ and $S$; (b) $A$ believes $\phi_{2 B}$ is only available to $A$ and $S$; (c) $A$ believes $m_{A}$ is only available to $A$ and $B$; (d) $B$ believes $m_{A}$ is only available to $A$ and $B$; (e) $A$ believes $m_{B}$ is only available to $A$ and $B$; (f) $B$ believes $m_{B}$ is only available to $A$ and $B$.

that the secrecy of $\phi_{2}$ is not required. What is important is to ensure that $\phi_{2}$ is provided afresh by a legal server. Therefore, the good-key security analysis that proves the secrecy, authenticity and freshness of $\phi_{2}$ is more than enough to ensure the correctness of the statement.

Next, the security analysis is performed in the mutual authentication phase of the proposed protocol. The following two properties are expected to be satisfied by the protocol:

1) Authentication of $A$ to $B: B$ believes $A$ is the correct device with its challenge $C_{A, B}$ and mask $\phi_{1}$ recorded in $B$. In this case, a honest device $B$ can correctly retrieve the device's PUF secret $P_{1 A}$ of $A$, i.e., $\tilde{P}_{1 A}=P_{1 A}$.
Therefore, for the honest $A$ and $B$, we have $A \mid \equiv$ $A \stackrel{P_{1 A}}{\longleftrightarrow} B$ and $B \mid \equiv A \stackrel{P_{1 A}}{\longleftrightarrow} B$.

2) Authentication of $B$ to $A$ : $A$ believes $B$ is the correct device with its helper information $C_{A, B}$ and mask $\phi_{2}$ recorded in $A$. In this case, a honest device $A$ can correctly retrieve the device's PUF secret $P_{2 B}$ of $B$, i.e., $\tilde{P}_{2 B}=P_{2 B}$. Therefore, for the honest $A$ and $B$, we have $A \mid \equiv A \stackrel{P_{2 B}}{\longleftrightarrow} B$ and $B \mid \equiv A \stackrel{P_{2 B}}{\longleftrightarrow} B$.

Mutual authentication can be established if only the authorized prover can send out the correct message digests of $m_{A}$ and $m_{B}$ (in Fig. 4), that match the ones calculated by their corresponding verifier. In other words, $m_{A}$ and 
$m_{B}$ should only be made available to the honest IoT peer nodes, $A$ and $B$. Therefore, the protocol's expected goal is: $m_{A}$ and $m_{B}$ are good shared keys between $A$ and $B$, i.e., $A\left|\equiv A \stackrel{m_{A}}{\longleftrightarrow} B, B\right| \equiv A \stackrel{m_{A}}{\longrightarrow} B, A \mid \equiv A \stackrel{m_{B}}{\longrightarrow} B$ and $B \mid \equiv A \stackrel{m_{B}}{\longrightarrow} B$.

According to the idealization rules of the MB logic, the protocol is firstly idealized as follows:

- $M s g_{1}: A \longrightarrow B: I D_{A}, T_{1}$

- $M s g_{2}: B \longrightarrow A: I D_{A}, E N C\left(m_{A} \Re T_{1}, P_{1 A}\right)$

- $M s g_{4}: B \longrightarrow A: I D_{B}, T_{2}$

- $M s_{5}: A \longrightarrow B: I D_{B}, E N C\left(m_{B} \Re T_{2}, P_{2 B}\right)$

The security analysis using the MB logic is shown in Figs. 5(c)-(f). To prove the correctness of $A \mid \equiv A \stackrel{m_{A}}{\longrightarrow} B$, the good key rule (6) can be applied first, which leads to two pre-conditions, $A\left|\equiv\{A, B\}^{c} \triangleleft\right| \mid m_{A}$ and $A \mid \equiv \#\left(m_{A}\right)$. Next, the fresh rule (5) is applied to prove $A \mid \equiv \#\left(m_{A}\right)$. Its correctness can be ensured if $A$ believes the freshness of $T_{1}$ and has received $m_{A}$ together with it. The correctness of $A \mid \equiv\{A, B\}^{c} \triangleleft \| m_{A}$ requires the proof of $A$ trusts $B$ on the condition that $m_{A}$ is only available to $A$ and $B$ according to the super-principal rule (4). To prove $A|\equiv B| \equiv\{A, B\}^{C} \triangleleft \| m_{A}$, the derived rule (8) is applied to obtain the three assumptions that: 1) $A|\equiv B| \equiv A \underset{P_{1 A}}{\stackrel{P_{1 A}}{\longrightarrow}} B$; 2) $A|\equiv B| \equiv\{B\}^{C} \triangleleft|| m_{A}$; and 3) $A|\equiv B| \sim m_{A}$. Assumptions 1) and 3) can be further proved with the nonce-verification rule (3) and the authentication rule (1). If the leaves of the tree (Fig. 5(c)), i.e., the initial beliefs, are reasonable, the fact that $A \mid \equiv A \stackrel{m_{A}}{\longrightarrow} B$ can be established. Similarly, the good-key rule and the confidentiality rule can be applied to obtain the initial beliefs for $B \mid \equiv A \stackrel{m_{A}}{\longleftrightarrow} B$ to be correct. For the two statements to be true, the initial beliefs derived are listed below:

1) $A \mid \equiv A \stackrel{P_{A A}}{\longleftrightarrow} B$ and $B \mid \equiv A \stackrel{P_{1 A}}{\longrightarrow} B$

2) $A \mid \equiv \#\left(T_{1}\right)$ and $B \mid \equiv \#\left(m_{A}\right)$

3) $A \mid \equiv \sup (B)$

4) $B \mid \equiv\{B\}^{C} \triangleleft \| m_{A}$

5) $A|\equiv B| \equiv\{B\}^{C} \triangleleft \| m_{A}$

6) $A \triangleleft m_{A} \Re T_{1}$

7) $A \stackrel{P_{1 A}}{\triangleleft} m_{A}$

8) $B \stackrel{P_{1 A}}{\sim} m_{A}$

Since $B$ is the super-principal for its hashed response $P_{1 B}$ and its generated $n_{A}, B$ is also the super-principal for $m_{A}$ as $m_{A}=P_{1 B} \oplus n_{A}$. Besides, $T_{1}$ and $m_{A}$ are freshly generated each time by $A$ and $B$. Therefore, the initial beliefs 1) to 5) are well satisfied. The remaining beliefs 6) to 8) are also reasonable as indicated by $\mathrm{Msg}_{2}$. As shown in Figs. $5(\mathrm{e})$ and (f), similar analysis can be conducted to prove the authentication of $B$ to $A$. The resulting initial assumptions and communication events are listed below:

1) $A \mid \equiv A \stackrel{P_{2 B}}{\longleftrightarrow} B$ and $B \mid \equiv A \stackrel{P_{2 B}}{\longleftrightarrow} B$

2) $B \mid \equiv \#\left(T_{2}\right)$ and $A \mid \equiv \#\left(m_{B}\right)$

3) $B \mid \equiv \sup (A)$

4) $A \mid \equiv\{A\}^{C} \triangleleft \| m_{B}$

5) $B|\equiv A| \equiv\{A\}^{C} \triangleleft \| m_{B}$

6) $B \triangleleft m_{B} \Re T_{2}$

7) $B \stackrel{P_{2 B}}{\triangleleft} m_{B}$

8) $A \mid \sim m_{B}$
By similar arguments and logical theory, all the initial beliefs are reasonable. Therefore, $m_{A}$ and $m_{B}$ are fresh in each authentication process and are only available to the honest principals, $A$ and $B$. Hence, according to the MB logic, mutual authentication between $A$ and $B$ is secure.

\subsection{ProVerif Analysis}

ProVerif [15] uses the symbolic, Dolev-Yao model [27] of cryptography for analysis. To be more specific, it treats the cryptographic primitives as black boxes, and the messages are viewed as terms on these primitives. The attacker is able to compute the messages but the computation is restricted to the use of only these primitives. Besides, it can intercept, modify, delete and inject messages sent on the network. ProVerif is sound in providing correspondence, reachability and observational equivalence proofs [28]. The proposed protocol introduced in Sec. 3 is translated into ProVerif scripts and arbitrarily many number of sessions and message space are simulated between protocol entities.

\subsubsection{Mutual Authentication and Various Attacks}

One objective of the proposed protocol is to ensure that the mutual authentication between two IoT peer nodes is valid and secure. The provenance proof of the mutual authentication also indicates the resistance of the protocol against replay and MITM attacks. To test this security property, the following events are declared:

1) event begin $A$ param(host $A$, host $B$ )

2) event endAparam(host $A$, hostB)

3) event beginBparam(host $B$, host $A$ )

4) event endBparam(host $B$, host $A$ ).

5) event begin $A f u l l(h o s t A$, host $B$, keyA, keyB).

6) event end $A$ full(host $A$, host $B$, key $A$, keyB).

7) event beginBfull(host $B$, host $A$, key $A$, keyB).

8) event endBfull(host $B$, host $A$, keyA, keyB).

Two different levels of authentication are used. The first four events prove the agreement on the host names only. "event beginAparam(hostA, hostB)" means hostB has commenced a run of the protocol with hostA and "event endAparam(hostA, hostB)" means hostA believes she has successfully completed the protocol with host $\mathrm{B}$, and vice versa for "event beginBparam(host $B$, host $A$ )" and "event endBparam(hostB, hostA)". The events that end with "full" are used for proving the agreement on all parameters of the protocol. For example, "event beginAfull(hostA, hostB, keyA, keyB)" denotes the intention of hostB to launch the protocol with hostA with the shared secret keys "keyA" and "keyB", and "event endAfull(hostA, hostB, keyA, keyB)" means hostA believes she has successfully completed the protocol with hostB with the declared shared secret keys.

If the mutual authentication is established, the following correspondence assertions should hold:

1) inj-event(endAparam(hostA,hostB))

$\Rightarrow$ inj-event(beginAparam(hostA,hostB))

which means if host $A$ reaches the end of the protocol and she believes she has completed the protocol with hostB, then hostB should have started a session with hostA. The relationship is injective. Similar implication holds for the following assertions. 
2) inj-event(endBparam(hostB,hostA)) $\Rightarrow$ inj-event(beginBparam(hostB,hostA))

3) inj-event(endAfull(host $A$,hostB,keyA,keyB)) $\Rightarrow$ inj-event(beginAfull(hostA,hostB,keyA,keyB))

4) inj-event(endBfull(hostB,host $A$, keyA,keyB)) $\Rightarrow$ inj-event(beginBfull(hostB,hostA,keyA,keyB))

\subsubsection{Reachability and Secrecy}

The other objective of the proposed protocol is to evaluate the reachability and secrecy of the shared secrets between the two IoT peer nodes. Reachability evaluation is a basic capability of ProVerif, which tests whether the term under test is reachable to an attacker. In the proposed protocol, the real secrets are the PUF responses of the two IoT devices, i.e., $R_{A}$ and $R_{B}$, to a selected challenge $C_{A, B}$. As the session keys established are the message digests of the PUF responses, if the session keys $\left(P_{2 A}\right.$ or $\left.P_{2 B}\right)$ are kept secret, the underlying PUF responses $\left(R_{A}\right.$ and $\left.R_{B}\right)$ will remain secret. Therefore, to test the reachability and secrecy of the PUF's CRP in the protocol, we only need to confirm the secrecy of the session keys. In our experiments, the following queries are included to test this property:

query attacker(messageA); attacker(messageB).

where "message $A$ " ("message $B$ ") is a free name sent by device $B(A)$ in encrypted form with the encryption key being $P_{2 A}$ $\left(P_{2 B}\right)$ over a public channel after the mutual authentication.

\subsubsection{Observational Equivalence}

In addition to the above correspondence and reachability proofs, four typical classes of observational equivalence $(O E)$ are also evaluated using ProVerif:

1) Strong secrecy:

This query evaluates if an attacker is able to distinguish when the secrets change. By denoting

\section{noninterf messageA; messageB}

the secrecy of the transmitted "messageA", "messageB" encrypted by the established session key $P_{2 A}$ or $P_{2 B}$ can be proved.

2) Off-line guessing attacks:

If the secrets are weak with low entropy, it may be successfully guessed by the attackers without further interaction with the protocol. The absence of off-line guessing attacks against "message $A$ " and "messageB" can be evaluated by the query:

\section{weaksecret messageA; messageB}

3) $O E$ between processes that differ only by terms:

This query tests the equivalence between two processes that have the same structure but different terms, which can be encoded by two single "biprocesses":

$$
\begin{aligned}
& \text { choice[ENC(messageA, } P_{2 A} \text { ), ENC(messageA, } \\
& \text { randomA))] } \\
& \text { choice[ENC(messageB, } P_{2 B} \text { ), ENC(messageB, } \\
& \text { randomB))] }
\end{aligned}
$$

where the construct "choice[term1,term2]" is used to represent the two processes with different terms while random $A$ and random $B$ are freshly generated random values.
4) Real-or-random secrecy:

This query evaluates whether the secrets are indistinguishable from the fresh random values. For example,

$$
\begin{aligned}
& \text { choice }\left[P_{2 A},\right. \text { randomA] } \\
& \text { choice }\left[P_{2 B},\right. \text { randomB] }
\end{aligned}
$$

As shown in Fig. 6, the proposed protocol has successfully achieved mutual authentication (correspondence assertion) and proved the secrecy of the shared keys (reachability) as well as the abovementioned four classes of observational equivalence.

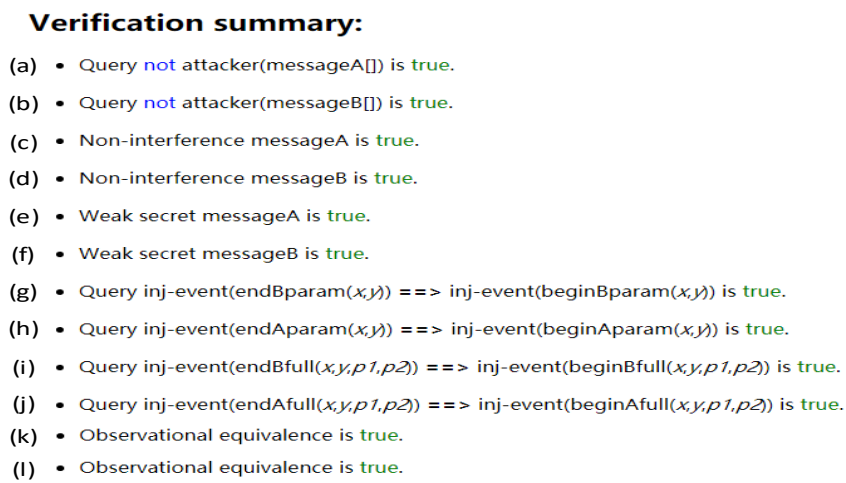

Fig. 6. ProVerif outputs. (a-b): Reachability proof; (c-d): Strong secrecy proof; (e-f): The absence of off-line guessing attacks proof; $(\mathrm{g}-\mathrm{j})$ : Mutual authentication proof; $(\mathrm{k}-\mathrm{l})$ : $O E$ between processes that differ only by terms and real-or-random secrecy proof.

\section{Prototype ANd Comparison}

\subsection{Hardware Implementation}

Fig. 7 shows the prototype implementation of our proposed system. Two Avnet Ultra96-V2 boards are used as the IoT endpoints. Ultra96-V2 is an ARM-based, Xilinx Zynq UltraScale $+{ }^{T M}$ MPSoC ZU3EG A484 development board. It supports PYNQ for Xilinx FPGA device, which means that Python language and libraries are provided for the application development to benefit from both the FPGA programmable logic and ARM microprocessor. The IoT endpoints are assumed to be in the same local area network (LAN). The LAN is created by a router but the router does not participate in the proposed scheme. A personal computer (PC) is used as the local server. The involvement of the local server is only in the enrolment and update phase as described in Sec. 3.

Any PUF with good uniqueness and randomness to minimize the probability of response collision or correlation that may be exploited by the attackers can be embedded into the IoT devices. The PUF can be flexibly selected to meet the device resource or any specific application requirements. A highly reliable PUF will relax the error correction requirement. Though PUF with near perfect reliability has been proposed in the literature [29], without loss of generality, we assume that the PUFs embedded in the devices have high but not perfect reliability. Therefore, ECC is required as the PUF responses are used for key generation in the proposed scheme. In our experiment, the reliable and modelling resistant Arbiter PUF from [30] is chosen for the implementation on the FPGA. The PUF overlay is then loaded into the 


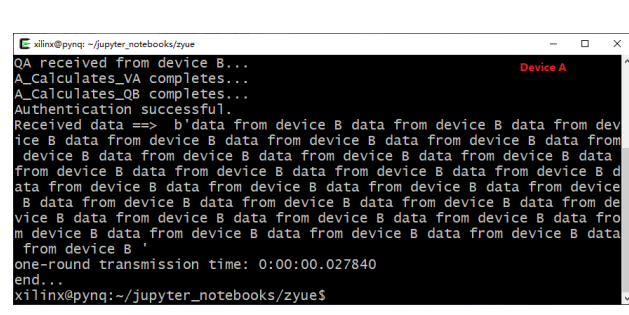

Output in device A's side in the authentication process
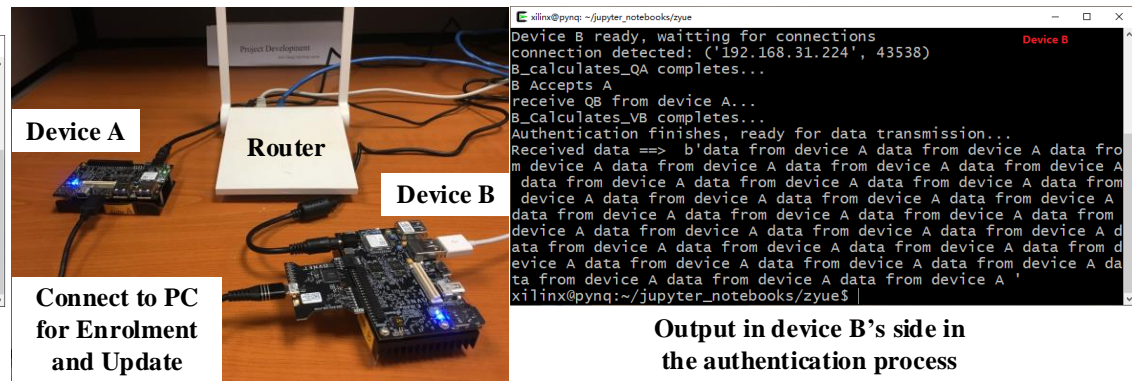

Fig. 7. Experimental set up and device $A$ and $B$ 's output in the mutual authentication and key exchange phase.

TABLE 3

Comparison with Existing PUF-based Protocols without CRP Storage

\begin{tabular}{c|c|c|c}
\hline \multirow{2}{*}{ Protocols } & \multicolumn{2}{|c|}{ Computational Complexity } & \multirow{2}{*}{ NVM Secret Free } \\
\cline { 2 - 5 } & $1 N_{H}+5 N_{U M A C}+2 N_{P U F}+3 N_{E n c}$ & Third Party* & Yes \\
\hline JIoT2017 [19] & $4 N_{P U F}$ & $2 N_{H}+4 N_{U M A C}+4 N_{E n c}$ (Server) & No \\
\hline TDSC2018 [13] & $9 N_{H}+1 N_{P U F}+1 N_{E C C}+3 N_{P A}+2 N_{P M}+1 N_{B P}$ & $24 N_{H}+10 N_{P A}+4 N_{P M}+2 N_{B P}$ (Verifier), -(SAP) & Yes \\
\hline Elsevier2019 [18] & $6 N_{H}+1 N_{P U F}+1 N_{E C C}+3 N_{P A}+7 N_{P M}$ & -(Authentication service) & Yes \\
\hline JSEN2021 [20] & $4 N_{H}+1 N_{P U F}+1 N_{E C C}+2 N_{E n c}$ & - & Yes \\
\hline This Work & Single Node & & \\
\hline
\end{tabular}

*: In this column, "(third party name)" means the third party doesn't have to perform computation but serves as an authentication helper data provider; '-' means no third party is needed.

ARM core for system design. The remaining cryptographic modules such as ECC and hash are directly imported from Python libraries. For demo purpose, the proposed protocol is designed on top of the network socket. The outputs of devices $A$ and $B$ in the authentication process are indicated in Fig. 7. It shows that the proposed mutual authentication and key exchange can be successfully conducted. The one-round transmission time, i.e., from the time the device $A$ prepares the encrypted data (the puzzle) for transmission to the time $A$ receives the encrypted data (the proof) from the device $B$, is only $27.8 \mathrm{~ms}$. This latency is considered low for the device and communication link we used for this demonstration. As only public information, i.e., device identity, challenge, ECC helper data and authentication mask, are stored in the device, the storage and security requirements in the device side are also low. As in the experiment, one such record is only 78 bytes if the device identity is presented by its IP address and a 128-bit PUF and SHA256 are used.

\subsection{Comparison With Existing Works}

There are only limited existing works [13], [18], [19], [20] that are able to achieve PUF-based device to device authentication without the need to store the CRPs in the verifier's side. In this section, only the related protocols in the existing works are compared, i.e., protocols in Fig. 3 of [13], [19] and [20], the BC-PHEMAP protocol in [18], and our proposed mutual authentication and key-exchange protocol presented in Fig. 4. The computational complexity both on the side of a single device node and that of a third party (if any) are listed and compared. Another important factor for protocol comparison is whether a locally stored device key is required (NVM secret free). Let $N_{H}, N_{U M A C}$,
$N_{P U F}, N_{E C C}, N_{E n c}, N_{P A}, N_{P M}$ and $N_{B P}$ denote the numbers of rounds of hash function, message authentication code based on universal hashing (UMAC), PUF, error correction code for PUF, encryption and decryption, point addition over elliptic curve, point multiplication over elliptic curve and bilinear pairing, respectively. Since the proposed protocol does not dictate the type of cryptographic modules such as PUF and hash as long as they meet the required functionality with acceptable implementation cost, Table 3 only lists the names of the cryptographic modules for a fair comparison. Similarly, for an implementation platform independent comparison of computational complexity, the number of rounds for each required operation is listed and compared. Trivial operations like concatenation or bitwise exclusive OR are omitted without impacting the relative accuracy of the comparison.

As shown in Table 3, the scheme [13] is the only one that requires a locally stored key and the three schemes [13], [18], [19] all require a third party to complete the mutual authentication between devices. The schemes [13] and [20] have the highest computational complexity among all as they both require public cryptography over elliptic curve for the protocol execution. The scheme [13] requires one bilinear pairing operation and two point multiplications to be performed in each device side while the work [20] requires seven rounds of point multiplications at each device side. A point multiplication over elliptic curve secp256r1 consumes around $112.34 \mathrm{~ms}$ on TI's CC2538 SoC (SHA256 only consumes around $0.061 \mathrm{~ms}$ in the same platform) [20]. As for bilinear pairing, it is widely regarded as an expensive operation for terminal devices [31]. The scheme of [18] is lightweight but its practicality is severely constrained by 
the PUF reliability problem. Besides, as analyzed in Sec. 2.2, it is also vulnerable to modelling attacks. As UMAC has similar computational complexity as hash operation [19], the computational complexity of the scheme of [19] can be approximated by $6 N_{H}+2 N_{P U F}+3 N_{E n c}$. To deal with the PUF reliability problem of this work, $2 N_{E C C}$ has to be added. Therefore, the overall complexity of [19] is estimated to be higher than our proposed scheme in addition to the need of a trust server. Besides the high performance, privacy and security achievable with typical device-side components, our proposed protocol outperforms those existing schemes in terms of achieving lost-cost direct P2P connection without the need for a secret key to be stored locally in the device.

\section{Conclusion}

This paper presents a PUF-based protocol for direct mutual authentication, automatic key-exchange and direct data transmission between IoT end devices. The proposed protocol overcomes the PUF's bottleneck in providing direct mutual authentication between resource-constrained devices without going through a proxy server. The requirement to store and safekeep a database of all provers' CRPs in the verifier's local memory is not only difficult to be satisfied by the resource-limited IoT devices, but the locally stored secret is also vulnerable to hardware attacks due to the accessibility of IoT endpoint devices. This security problem is solved by creating and storing the authentication mask data that do not need to be kept private in the device side. A new and important side benefit of the proposed protocol is that secure key-exchange between the two endpoints is directly established upon successful mutual authentication with neither additional flight message transfer nor complex public-key algorithm. Formal MB logic analysis and ProVerif simulation have been performed to prove the security soundness of the proposed protocol. The hardware cost and computational complexity are reasonably low in comparison with the existing PUF-based CRP-storage-free authentication protocols.

\section{References}

[1] I. HBSCHMANN, "P2P IoT vs. Database-driven IoT Which to choose?" Nov. 27 2018. [Online]. Available: https://www.nabto.com/p2p-vs-database-iot/

[2] Y. Zheng, Y. Cao, and C. H. Chang, "UDhashing: Physical unclonable function based user-device hash for endpoint authentication," IEEE Trans. Industrial Electron., vol. 66, no. 12, pp. 9559-9570, Dec. 2019.

[3] Y. Zheng, Y. Cao, and C. H. Chang, "A PUF-based data-device hash for tampered image detection and source camera identification," IEEE Trans. Inf. Forensics, Security, vol. 15, pp. 620-634, Jul. 2019.

[4] J. R. Wallrabenstein, "Practical and secure IoT device authentication using physical unclonable functions," in Proc. 2016 IEEE 4th Int. Conf. Future Internet Things Cloud (FiCloud). Vienna, Austria: IEEE, Aug. 2016, pp. 99-106.

[5] T. Idriss and M. Bayoumi, "Lightweight highly secure PUF protocol for mutual authentication and secret message exchange," in Proc. 2017 IEEE Int. Conf. RFID Technol. Appl. (RFID-TA). Warsaw, Poland: IEEE, 2017, pp. 214-219.

[6] P. Gope and B. Sikdar, "Lightweight and privacy-preserving twofactor authentication scheme for IoT devices," IEEE Internet Things J., vol. 6, no. 1, pp. 580-589, Feb. 2018.
[7] C. H. Chang, Y. Zheng, and L. Zhang, "A retrospective and a look forward: Fifteen years of physical unclonable function advancement," IEEE Circuits Syst. Mag., vol. 17, no. 3, pp. 32-62, Aug. 2017.

[8] S. P. Skorobogatov, "Semi-invasive attacks - A new approach to hardware security analysis," University of Cambridge, Tech. Rep. 630, Apr. 2005.

[9] F. Courbon, S. Skorobogatov, and C. Woods, "Reverse engineering flash EEPROM memories using scanning electron microscopy," in Proc. Int. Conf. Smart Card Research Adv. Appl. Cannes, France: Springer, Nov. 2016, pp. 57-72.

[10] C. Helfmeier, D. Nedospasov, C. Tarnovsky, J. S. Krissler, C. Boit et al., "Breaking and entering through the silicon," in Proc. 2013 ACM SIGSAC conf. Comput. Commun. Security. Berlin, Germany: ACM, Nov. 2013, pp. 733-744.

[11] N. F. Ghalaty, B. Yuce, M. Taha, and P. Schaumont, "Differential fault intensity analysis," in 2014 Workshop Fault Diagnosis Tolerance in Cryptography. Busan, South Korea: IEEE, Sept. 2014, pp. 49-58.

[12] Y. Zheng and C. Chang, "Secure mutual authentication and keyexchange protocol between PUF-embedded IoT endpoints," in 2021 IEEE Int. Symp. Circuits Syst. (ISCAS), Daegu, Korea, May 2021, pp. 1-4.

[13] U. Chatterjee, V. Govindan, R. Sadhukhan, D. Mukhopadhyay, R. S. Chakraborty et al., "Building PUF based authentication and key exchange protocol for IoT without explicit CRPs in verifier database," IEEE Trans. Dependable Secure Comput., vol. 16, no. 3, pp. 424-437, May-Jun. 2018.

[14] W. Mao and C. Boyd, "Towards formal analysis of security protocols," in Proc. Computer Security Foundations Workshop VI, Jun. 1993, pp. 147-158.

[15] B. Blanchet, B. Smyth, V. Cheval, and M. Sylvestre, "Proverif 2.02: Automatic cryptographic protocol verifier, user manual and tutorial," pp. 01-129, Jul. 2020.

[16] Grayshift, "GrayKey: Extract evidence from encrypted mobile devices." [Online]. Available: https://www.grayshift.com/

[17] Cellebrite, "Cellebrite UFED: The industry standard for accessing digital device data." [Online]. Available: https://www.cellebrite.com/en/ufed/

[18] M. Barbareschi, A. De Benedictis, E. La Montagna, A. Mazzeo, and N. Mazzocca, "A PUF-based mutual authentication scheme for cloud-edges IoT systems," Future Generation Comput. Syst., vol. 101, pp. 246-261, Dec. 2019.

[19] M. N. Aman, K. C. Chua, and B. Sikdar, "Mutual authentication in IoT systems using physical unclonable functions," IEEE Internet Things J., vol. 4, no. 5, pp. 1327-1340, Oct. 2017.

[20] S. Li, T. Zhang, B. Yu, and K. He, "A provably secure and practical PUF-based end-to-end mutual authentication and key exchange protocol for IoT," IEEE Sensors J., vol. 21, no. 4, Feb. 2021.

[21] M. Burrows, M. Abadi, and R. M. Needham, "A logic of authentication," Proc. Royal Society of London. A. Mathematical and Physical Sciences, vol. 426, no. 1871, pp. 233-271, Feb. 1989.

[22] M. E. R. Baraa Tareq Hammad, Norziana Jamil and M. R. Z'aba, "A survey of lightweight cryptographic hash function," Int. J. Scientific Eng. Research, vol. 8, pp. 806-814, Jul. 2017.

[23] H. Kaul, M. Anders, S. Mathew, V. Suresh, S. Satpathy, A. Agarwal, S. Hsu, and R. Krishnamurthy, "Ultra-lightweight 548-1080 gate 166gbps/w12.6tbps/w SIMON 32/64 cipher accelerators for IoT in 14nm tri-gate CMOS," in Proc. 2018 IEEE Asian Solid-State Circuits Conf. (A-SSCC), Taiwan, Nov 2018, pp. 1-4.

[24] A. Houghton, Error coding for engineers. Springer Science \& Business Media, 2001, vol. 641.

[25] A. Aghaie and A. Moradi, "TI-PUF: Toward side-channel resistant physical unclonable functions," IEEE Trans. Information Forensics and Security, vol. 15, pp. 3470-3481, Apr. 2020.

[26] Y. Nozaki and M. Yoshikawa, "Countermeasure of lightweight physical unclonable function against side-channel attack," in 2019 Cybersecurity and Cyberforensics Conference (CCC). IEEE, May 2019, pp. 30-34.

[27] D. Dolev and A. Yao, "On the security of public key protocols," IEEE Trans. Information Theory, vol. 29, no. 2, pp. 198-208, Mar. 1983.

[28] S. Malladi and B. Blanchet, "ProVerif web interface." [Online]. Available: http:/ / proverif16.paris.inria.fr/index.php

[29] D. Jeon et al., "A Physical Unclonable Function With Bit Error Rate $<2.3 \times 10^{-8}$ Based on Contact Formation Probability Without Error Correction Code," IEEE J. Solid-State Circuits, vol. 55, no. 3, pp. 805-816, Nov. 2019. 
[30] S. S. Zalivaka, A. A. Ivaniuk, and C. H. Chang, "Reliable and modeling attack resistant authentication of arbiter PUF in FPGA implementation with trinary quadruple response," IEEE Trans. Information Forensics and Security, vol. 14, no. 4, pp. 1109-1123, Apr. 2018.

[31] K. Sowjanya, M. Dasgupta, S. Ray, and M. S. Obaidat, "An efficient elliptic curve cryptography-based without pairing KPABE for Internet of Things," IEEE Systems Journal, vol. 14, pp. 2154-2163, Jun. 2019.

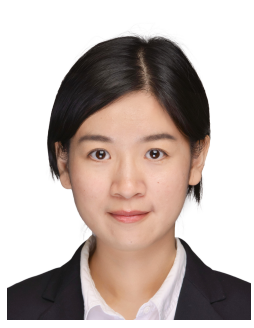

Yue Zheng (S'15-M'20) received the B. Eng. Degree in School of Communication and Information Engineering from Shanghai University (SHU, China), and Ph.D. degree in School of Electrical and Electronic Engineering, Nanyang Technological University (NTU, Singapore), in 2015 and 2020, respectively. She was a visiting scholar to Kyoto University between March to June 2019 and she is currently a researcher fellow in NTU. Her area of research includes hardware security, physical unclonable function (PUF) for device fingerprinting and secret key generation, design of PUF-based security protocols. Dr. Zheng serves as the review committee member and special session chair of 2021 IEEE International Symposium on Circuits and Systems (ISCAS 2021), and the track chair of the $29^{\text {th }}$ IFIP/IEEE International Conference on Very Large Scale Integration (VLSI-SoC 2021).

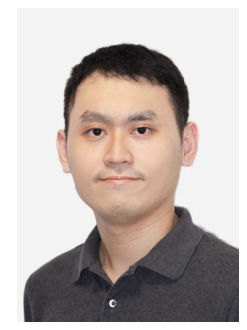

Wenye Liu (S'17) received the B.S. degree in microelectronics from Shenzhen University, China, in 2014, the B.S. degree in physics from Umea University, Sweden, in 2014, and the M.S. degree in IC design engineering from Hong Kong University of Science and Technology. He is currently pursuing the Ph.D degree with the School of Electrical and Electronic Engineering, Nanyang Technological University, Singapore. His current research interests include hardware security, machine learning accelerator and fault

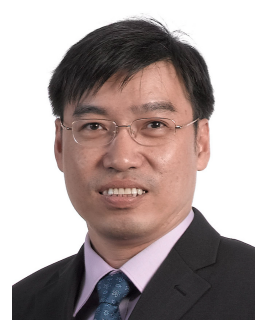

Chip-Hong Chang (S'92-M'98-SM'03-F'18) received the B.Eng. (Hons.) degree from the National University of Singapore in 1989, and the M. Eng. and Ph.D. degrees from Nanyang Technological University (NTU) of Singapore in 1993 and 1998, respectively. $\mathrm{He}$ is an Associate Professor of the School of Electrical and Electronic Engineering (EEE) of NTU. $\mathrm{He}$ held joint appointments with the university as Assistant Chair of Alumni from 2008 to 2014, Deputy Director of the Center for High Performance Embedded Systems from 2000 to 2011, and Program Director of the Center for Integrated Circuits and Systems from 2003 to 2009. He has coedited 4 books, published 13 book chapters, more than 100 international journal papers (more than 70 are in IEEE) and more than 180 refereed international conference papers (mostly in IEEE), and delivered over 40 colloquia. His current research interests include hardware security, unconventional number systems, and low-power and fault-tolerant digital signal processing algorithms and architectures.

Dr. Chang currently serves as the Senior Area Editor of IEEE Transactions on Information Forensic and Security, and Associate Editor of the IEEE Transactions on Circuits and Systems-I and IEEE Transactions on Very Large Scale Integration (VLSI) Systems. He also served as the Associate Editor of the IEEE Transactions on Information Forensic and Security and IEEE Transactions on Computer-Aided Design of Integrated Circuits and Systems from 2016 to 2019, IEEE Access from 2013 to 2019, IEEE Transactions on Circuits and SystemsI from 2010 to 2013, Integration, the VLSI Journal from 2013 to 2015, Springer Journal of Hardware and System Security from 2016 to 2020 and Microelectronics Journal from 2014 to 2020. He guest edited around 10 special issues and served in the organizing and technical program committee of more than 60 international conferences (mostly IEEE). He is an IEEE Fellow, IET Fellow, and 2018-2019 Distinguished Lecturer of IEEE Circuits and Systems Society.

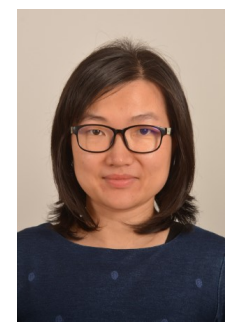

Chongyan Gu (S'14-M'16) received the Ph.D. degree from Queen's University Belfast, Belfast, U.K., in 2016. She is currently a Lecturer (Assistant Professor) in the School of EEECS at Queen's University Belfast, and a member of Center for Secure Information Technologies (CSIT) with in the Institute of Electronics Communications and Information Technologies (ECIT). Dr. Gu is an expert in hardware security. Her research into physical unclonable function (PUF) has been utilised as part of a security architecture for electronic vehicle (EV) charging systems, licensed by LG-CNS, South Korea, and was also licensed for evaluation by Thales, U.K.. Her team was the overall winner of INVENT 2015, a competition to accelerate the commercialisation of innovative ideas. She is a technical committee member of International Symposium on Circuits and Systems (ISCAS). She was invited to publish a paper in the Proceedings of the IEEE (IF: ¿10). She has co-authored two research book chapters published by IET in 2016 and Springer in 2018, respectively. She has successfully organised two conference special sessions (IEEE APCCAS in 2018 and ACM GLSVLSI in 2020). She was invited to give tutorial/talks to international conferences, such as, IEEE ASPDAC 2020 on the topic of practical PUF design on FPGA. Her current research interests include PUFs, security in/for approximate computing, true random number generator (TRNGs), hardware Trojan detection and machine learning attacks. 